\title{
306 DECR2 REGULATES TUMOR CELL FERROPTOSIS AND IS REQUIRED FOR IMMUNOTHERAPY EFFICACY
}

Shuyin Li*, Thomas Gajewski, Shuyin Li, Emily Higgs. University of Chicago, Chicago, IL, USA

Background Checkpoint blockade therapies have transformed the landscape of cancer care. Durable clinical responses have been observed in a subset of patients. However, many patients do not respond, and understanding the mechanisms underlying tumor resistance to checkpoint blockade drugs could potentially expand clinical benefit. We therefore performed an unbiased CRISPR library screen to identify novel gene alterations sufficient to mediate resistance to $\mathrm{T}$ cell-mediated killing. Methods B16.SIY cells were transduced with a genome-scale gRNA lentivirus to generate loss of function mutants. In vitroprimed CD8 + T cells isolated from 2C/Rag2-/- TCR transgenic mice specific for the SIY antigen were co-cultured with transduced B16.SIY tumor cells. Resistant cells were collected and targeted genes were identified by sequencing the gRNAs. The gene encoding Decr2, a peroxisomal 2,4-dienoyl-CoA reductase, was identified. To investigate the role of Decr2 in tumor growth and immune responses in vivo, the Decr2 knock-down or Decr2 overexpressed tumors were transplanted into C57BL/6 mice for studies of anti-PD-L1 efficacy and immune response analysis. Based on a hypothesized role in ferroptosis, RNA-seq was performed and lipid reactive oxygen species were quantified.

Results Decr2 mutants were relatively resistant to CD8 $+\mathrm{T}$ cell killing in vitro. In vivo, Decr2 knock-down tumors showed almost complete loss of anti-PD-L1 efficacy, without affecting early $\mathrm{T}$ cell priming or immune cell infiltration. However, tumor-infiltrating $\mathrm{T}$ cells failed to expand following anti-PD-L1 therapy in Decr2 knock-down tumors. RNA-seq analysis revealed upregulation of ferroptosis-related genes in Decr2-deficient tumor cells, and these tumor cells were relatively resistant to ferroptosis-inducers in vitro. Decr2 knockdown cells also showed defective induction of lipid ROS in response to CD8 + T cells in vitro, or when analyzed following anti-PD-L1 treatment ex vivo. Analysis of tumor RNA-seq data from human melanoma patients treated with checkpoint blockade revealed decreased Decr2 levels in non-responding patients.

Conclusions Our results identify tumor cell-expressed Decr2 as novel regulator of ferroptosis and implicate the process of ferroptosis as a major mechanism of immune-mediated tumor cell killing in vivo. Pharmacologic strategies to facilitate tumor cell ferroptosis could have therapeutic utility.

http://dx.doi.org/10.1136/jitc-2021-SITC2021.306 